\title{
L'ÉDUCATION MUSÉALE, UN RAPPORT AU SAVOIR
}

\begin{abstract}
Anik Meunier $^{1}$
Les musées sont le reflet des sociétés dans lesquelles ils se développent et prennent naissance, et en établir une définition unique implique souvent de les réduire à ce qu'ils ne sont pas nécessairement. Premier directeur de l'ICOM, de 1948 à 1965, George-Henri Rivière considérait que le musée devait poursuivre trois fonctions complémentaires : la recherche, la conservation et la présentation, qui justifient son existence en tant qu'institution sociale. Pour lui, l'interdépendance des trois fonctions du musée " [...] renforce cette nécessité d'équilibre et justifie l'existence du musée : les conservatoires et les dépôts assurent la préservation des objets, les universités font de la recherche, les centres culturels excellent dans l'animation et montent des expositions. Seul le musée assure simultanément ces différentes fonctions et enrichit ses activités de leurs relations mutuelles. "2 Cette idéologie transparaît nettement dans la première définition des musées adoptée par l'ICOM en 1951 : " Le mot musée désigne ici tout établissement permanent, administré dans l'intérêt général en vue de conserver, étudier, mettre en valeur par des moyens divers et essentiellement exposer pour la délec-

1 Professeure à l'Université du Québec à Montréal (UQAM). L'auteure tient à souligner la collaboration à ce texte de Charlène Bélanger et Aude Joly, toutes deux étudiantes au sein du programme de maîtrise en muséologie, respectivement à l'Université du Québec à Montréal (UQAM) et à l'Université de Montréal.

2 G.-H. RIVIÈRE, La muséologie, Dunod, 1989, pp. 52-53.
\end{abstract}

Recherches en communication, $\mathrm{n}^{\circ} 29$ (2008). 
tation et l'éducation du public un ensemble d'éléments de valeur culturelle : collections d'objets artistiques, historiques, scientifiques et techniques, jardins botaniques et zoologiques et aquariums ". Cette définition se modifie et, en 1974, l'ICOM en adopte une qui est encore maintenue actuellement : "Le musée est une institution permanente, sans but lucratif, au service de la société et de son développement, ouverte au public, et qui fait des recherches concernant les témoins matériels de l'homme et de son environnement, acquiert ceux-là, les conserve, les communique et notamment les expose à des fins d'études, d'éducation et de délectation ". ${ }^{1}$ Ainsi, les musées partagent des fonctions particulières qui les définissent en tant qu'institution. Selon les différents auteurs, celles-ci peuvent être regroupées sous trois, quatre ou cinq grandes fonctions. Van Mensch ${ }^{2}$ a développé un modèle, repris par Mairesse $^{3}$ qui comporte trois fonctions contenues dans la définition de l'ICOM : la préservation, la recherche et la communication, auxquelles s'ajoute une quatrième fonction, tout aussi essentielle à l'existence des musées : l'administration. Chacune de ces grandes fonctions comporte plusieurs composantes. La préservation concerne l'acquisition, l'aliénation, la conservation, la restauration et l'archivage. Quant à la recherche, il existe, selon Alexander, trois grands types de recherche au sein des institutions muséales : la recherche fondamentale, la recherche appliquée et la recherche sur les publics ${ }^{4}$. La fonction de communication concerne pour sa part les expositions, l'éducation, l'interprétation, l'animation, les publications et l'action culturelle. Enfin, de manière systémique et transversale puisqu'elle a trait tant aux fonctions de préservation, de recherche et de communication, l'administration relève notamment de la planification, des finances, du développement institutionnel, des ressources humaines et matérielles, des relations avec les partenaires, du service des communications et du marketing. Ces différentes fonctions s'articuleront de manière spécifique selon un ensemble de particularités relatives à l'institution muséale, dont ses orientations et son mandat; sa taille; son statut public ou privé.

1 Conseil international des musées (ICOM), Nouvelles de l'ICOM, Statuts de l'ICOM, article 2, paragraphe 1, vol. 57, no.2, 2004.

2 P. VAN MENSCH, Towards a methodology of museology, Ph.D Thesis, Univertisy of Zagreb, 1992.

3 F. MAIRESSE, Le musée, temple spectaculaire, Lyon, Presses universitaires de Lyon, 2002.

4 E.P. AlEXANDER, Museums in Motion, Nashville, T.N. : American Association for State and Local History, 1979. 
Les musées se sont constitués telles des entités définissant leurs champs de référence et leur rapport au savoir à travers des sociétés et des époques au sein des institutions qu'ils ont représentées. Toutefois, ils partagent ce domaine de légitimité avec d'autres institutions et structures sociales. Les diverses institutions de formation, dont l'école, sont de celles-là. Ainsi, au sein même des musées, se sont progressivement structurés et organisés des services dont l'intention poursuivie consiste à diffuser et à partager les diverses formes de savoir à l'intention du plus grand nombre et non plus uniquement aux experts et aux spécialistes. C'est ainsi que se développe un champ disciplinaire communément appelé l' "éducation muséale".

\section{L'éducation muséale, un domaine singulier de l'éducation et de la muséologie}

L'éducation muséale demeure une notion souvent floue, aux contours encore mal définis. Celle-ci détermine une expression en usage dans la langue française mais dérivée directement de la traduction de l'expression anglo-saxonne museum education. Elle recouvre les notions d'éducation et de musée. On sait d'entrée de jeu qu'il s'agit de deux notions larges et complexes à cerner. L'éducation peut être entendue comme la formation, l'initiation, l'instruction ou encore la pédagogie, mais peut aussi bien concerner la mise en œuvre des différents moyens visant à assurer la formation et le développement d'un être humain. Quant à la notion de musée, précisons d'office que par les différentes disciplines et champs de recherche et d'interventions auxquels il renvoie, le musée peut s'intéresser tant aux domaines des sciences, des arts, de l'histoire, de l'ethnologie, de l'archéologie qu'à des thématiques telles que l'environnement ou les sociétés. Il va sans dire que de pareilles préoccupations sont à la fois hétérogènes et nécessairement non consensuelles. Toutefois, il est possible, de manière très large et donc forcément réductrice, de cerner un dénominateur commun à l'ensemble des institutions muséales. Il s'agit du souci du visiteur qui apparaît dorénavant comme source centrale des préoccupations et souvent le moteur même des actions de démocratisation de la culture et du patrimoine à l'intention des diverses catégories de public.

Afin de légitimer les diverses actions dans le domaine de l'éducation muséale, on fait fréquemment appel au caractère complémentaire du lieu muséal pour favoriser l'approche sensible par les " vraies 
choses " que l'école ne peut proposer ou encore à l'originalité et au renouvellement des formes qu'adopte l'offre éducative en contexte muséal ${ }^{1}$. Pourtant, ni l'un ni l'autre de ces arguments ne suffisent à justifier l'approche éducative dans le domaine de la muséologie. La première assertion est de moins en moins valable puisque de plus en plus se développent - même s'ils sont mal connus - , des projets comme les " classes-musées " ou des " expositions à l'école ", qui viennent compléter l'enseignement théorique prodigué en classe ${ }^{2}$. C'est à la manière de la "leçon de chose " comme approche pédagogique que les collections intègrent parfois les classes et que les écoliers ont la possibilité d'appréhender et d'illustrer un concept scientifique ou historique à l'aide d'objets patrimoniaux, un peu à l'image de l'usage des collections des premiers musées universitaires, comme le Musée Ashmolean d'Oxford aux Etats-Unis, qui, dès 1683, agissait comme lieu de conservation et de recherche et mettait ses collections à la disposition des étudiants ${ }^{3}$. Rappelons aussi à titre d'exemple le musée commercial et industriel de la toute première école des HEC à Montréal. Consacré à la présentation de l'industrie et du commerce sous toutes leurs formes, le musée de l'École des HEC (1910-1956) a constitué un outil pédagogique précieux pour les étudiants de cette école ${ }^{4}$. Ainsi, des approches

1 D.F. CAMERON, “ Un point de vue : le musée considéré comme système de communication et les implications de ce système dans les programmes éducatifs muséaux ", in Vagues : une anthologie de la nouvelle muséologie, Collection Museologia/Muséologie nouvelle et expérimentation sociale. Mâcon, Éditions W \& Savigny-le-Temple, vol. 1, 1968, pp. 259-270.

2 Les classes-musées de la Havane à Cuba sont issues d'une question relativement simple à résoudre : le besoin de re-localiser les groupes d'élèves lors de la restauration du patrimoine " bâti ”, dont les écoles. En réponse à cette situation, la proposition consiste à déplacer les enseignants et leurs élèves en réaménageant leurs classes au sein d'un lieu culturel (musée, cathédrale, lieu historique) afin de poursuivre l'enseignement académique tel que proposé par les programmes d'études en vigueur à Cuba. Cette initiative, qui date d'une dizaine d'années, est née de la problématique de restauration qui nécessite l'évacuation temporaire des citoyens pendant la durée des travaux.

3 M. AllARD et C. DUfRESNE-TASSÉ, " Le développement au Canada des recherches en éducation muséale : essai de synthèse ", in A Challenge Met : the Definition and Recognition of the Field of Education / Un défi relevé : la définition et la reconnaissance du champ d'étude de l'éducation, Ottawa, Canadian Society for the Study of Education / Société canadienne pour l'étude de l'éducation, 1999, pp. 145-166.

4 Site du service des archives de HEC Montréal, A012 - Fonds du Musée commercial et industriel de Montréal. - 1910-1960. - 71,5 cm de documents textuels. - 222 
pédagogiques en lien avec les musées sont, selon certaines périodes historiques, plus ou moins mises de l'avant. Quant à la seconde proposition, soit le renouvellement des formes qu'adopte l'offre éducative en contexte muséal, même si elle s'avère intéressante, elle n'est que rarement originale, novatrice et renouvelée. On se trouve plutôt souvent face à une répétition d'approches qui ont fait plus ou moins bien leurs preuves, qui satisfont à peu près les visiteurs et les participants et qui atteignent à peu près les intentions éducatives poursuivies à l'intention des élèves-visiteurs. La lourdeur d'une démarche évaluative afin de connaître les retombées véritables des actions d'éducation est telle qu'il n'est pas toujours possible de l'intégrer à la pratique professionnelle des musées. De manière plus souple, les médiateurs qui agissent en face à face avec les visiteurs s'enquièrent de leur appréciation de la visite afin de se conforter dans leurs prestations et de s'assurer de la satisfaction des visiteurs à l'égard de leur participation aux diverses manifestations dans le musée. Ainsi, une certaine stabilité quant aux actions éducatives est maintenue, sans pour autant que l'on puisse être convaincu de leur portée réelle.

\section{Définir les formes de l'éducation muséale}

De nombreuses actions de communication et de diffusion, voire d'éducation, de la culture et du patrimoine sont conçues et développées par les différents acteurs de la muséologie. Les deux modes de communication privilégiés par lesquels ils s'adressent aux visiteurs sont le média exposition et/ou les programmes éducatifs développés au sein du musée et, idéalement, à partir des diverses ressources disponibles, notamment des collections.

Le champ disciplinaire de l'éducation muséale s'est progressivement constitué par le nombre important de recherches menées dans ce domaine vaste et complexe, notamment depuis les trente dernières années au Québec. Cependant, il n'est pas tout à fait logé à l'enseigne des préoccupations d'éducation qui renvoient au cadre scolaire et scientifique, mais regroupe plutôt les diverses actions réalisées et qui tendent à susciter des apprentissages, à conditionner des comportements

photographies http://www.hec.ca/archives/institutionnelles/A012.html (site consulté en mars 2008) 
sociaux, voire à provoquer des attitudes sociales ou à engendrer de nouveaux intérêts. Comme ces assertions peuvent être questionnées ou même réfutées, il faut aussi clairement rappeler que les formes d'éducation qui prennent place en contexte muséal sont souvent distinctes des approches préconisées à l'école. L'éducation muséale se rattache au champ de l'éducation non formelle et se définit en continuité ou en rupture, partielle ou totale des intentions du programme d'étude officiel des institutions de formation. À juste titre, les chercheurs se sont intéressés à documenter et analyser plusieurs approches qui tentent de cerner un ensemble de dimensions éducatives et circonscrire le rôle spécifique de l'éducation dans le domaine de la muséologie et plus particulièrement des actions d'éducation muséale.

L'analyse des types de projets pédagogiques ou des formations offertes aux enseignants ou aux élèves permet principalement d'établir la panoplie des types de relations entre l'école et le musée. Ainsi, quelques auteurs ont distingué des types de relation pouvant se développer entre l'école et le musée, dont l'harmonisation du musée et de l'école, la parascolarisation du musée et la déscolarisation du musée ${ }^{1}$; la prise en charge des missions de l'école par le musée ${ }^{2}$; et, plus récemment, la scolarisation du musée ${ }^{3}$.

L'harmonisation du musée et de l'école ${ }^{4}$ consiste ainsi en une relation qui s'établit entre l'école et le musée. Idéalement, elle se définit comme un partenariat qui respecte les missions, les rôles, les approches, les méthodes, les stratégies et les contenus d'apprentissage de chaque partenaire. Les musées qui favorisent ce type de relation avec l'école prennent en compte le contenu de leurs collections tout en favorisant l'adéquation de ces contenus avec celui des programmes d'études des écoles.

1 M. PAQUIN, La visite scolaire au musée, Cap Rouge, Les Presses Inter Universitaires, 1994, p. 26.

2 M.I. ORELLANA et I. DE LA JARA, “ L'émergence du partenariat scientifique écolemusée au Musée des enfants de Santiago du Chili ”, in Aster, numéro 29, vol. 41, 1999, pp. 41-60.

3 C. COHEN, Quand l'enfant devient visiteur : une nouvelle approche du partenariat École/Musée, L'Harmattan, Paris, 2002. 118 p.

4 M. PAQUIN, op. cit. 
Les musées qui œuvrent dans une optique de parascolarisation du musée ${ }^{1}$ considèrent que l'accueil des groupes scolaires doit être une priorité et font le choix d'offrir des programmes et des activités éducatives spécifiquement destinés aux groupes scolaires, mais qui ne sont pas nécessairement en lien avec les programmes d'études des écoles. Ce type d'offre convient parfaitement à certains enseignants ou à des écoles qui utilisent la sortie scolaire au musée pour rencontrer d'autres objectifs que ceux purement pédagogiques.

Les musées qui travaillent dans une optique de déscolarisation du musée ${ }^{2}$ refusent de considérer le public scolaire comme un public spécifique et réduisent les arrangements institutionnels avec l'école. Ainsi, les groupes scolaires participent aux mêmes activités éducatives que les autres publics du musée. Ces programmes se présentent généralement sous la forme de visites guidées ou de visites libres des expositions. Ces musées établissent une relation de déscolarisation vis-à-vis des groupes en provenance des écoles et ne développent pas précisément de programmes éducatifs à l'intention de ces publics.

Maria Isabel Orellana et Irene de la Jara ${ }^{3}$ mettent en évidence un quatrième type de relation école-musée qui consiste en la prise en charge des missions de l'école par le musée. Cette prise en charge est très fréquente dans le contexte des musées et des centres de sciences, non seulement en Amérique latine, mais également en Amérique du Nord et en Europe. En effet, les nouveaux programmes scolaires de la plupart des pays occidentaux demandent aujourd'hui aux enseignants d'enseigner les sciences et la technologie de manière obligatoire à tous les niveaux de l'école primaire. Ceux-ci sont souvent démunis face à cette nouvelle demande des autorités scolaires. Ils se tournent alors vers des ressources externes pour développer des outils et des stratégies d'apprentissage susceptibles de les soutenir dans leur tâche. Plusieurs enseignants choisissent même d'organiser des sorties scolaires au musée de sciences dans l'espoir que le musée pourra prendre en charge une partie du programme d'études et ainsi les décharger d'une partie de leur tâche. À l'intérieur de ce type d'ateliers pédagogiques qui touchent des sujets

1 Idem.

2 Ibidem.

3 M.I. ORELLANA et DE LA JARA, op. cit. 
directement en lien avec les programmes scolaires, mais difficilement abordables en classe, les musées mettent un éducateur scientifique à la disposition du groupe scolaire. Dans ce contexte, l'enseignant n'a pas de rôle précis à jouer, il observe les élèves et soutient l'éducateur dans la gestion disciplinaire du groupe. Au terme de l'atelier, on remet généralement à l'enseignant un document qui lui suggère des activités complémentaires à réaliser en classe avec ses élèves. Ainsi, le musée se substitue, totalement ou partiellement, à l'enseignant dans sa tâche d'enseigner les sciences et la technologie aux élèves de sa classe.

Cohen ${ }^{1}$ soutient que, historiquement, les tentatives d'harmonisation des relations entre l'école et le musée en France ont mené à la scolarisation du musée plutôt qu'à un réel partenariat dans le respect des finalités des deux institutions. Cette chercheure, avance trois raisons : l'utilisation des objets des collections à des fins d'illustration, la présence de nombreux enseignants à l'origine des services pédagogiques des musées et la conception d'outils et d'aides à la visite de type fiche-enquête. Le musée devient, dans cette perspective, une simple ressource au service de l'école et ce sont essentiellement les objectifs de l'activité et du projet scolaire qui déterminent les liens de complémentarité entre le musée et l'école. Le sens de la visite est défini par son seul rapport à une activité pédagogique réalisée en classe. La spécificité du musée devient secondaire. La relation qui s'établit entre l'école et le musée dans ces conditions ne constitue pas un véritable partenariat d'échange entre les deux institutions, mais place plutôt le musée au service de l'école. Le musée perd ainsi sa spécificité et est considéré comme un réservoir de matériel pédagogique par le milieu scolaire ${ }^{2}$.

Au-delà de ces tentatives d'analyses des types de projets pédagogiques ou des formations offertes aux enseignants ou aux élèves afin

1 C. COHEN, "Visite scolaire au musée : représentations d'enseignants en formation initiale en France et au Québec", in L'Accueil des publics scolaires dans les Museums, Aquariums, Jardins botaniques, Parcs zoologiques. Yves Girault, Paris, L'Harmattan, 2003, pp. 195-225.

2 Pour plus de détails quant à ces questions, voir C. BÉLANGER et A. MEUNIER, Vers une approche muséologique de la visite scolaire au musée : projet de formation à l'intention des futurs enseignants québécois, Les cahiers du Groupe de recherche sur l'éducation et les musées (GREM), Université du Québec à Montréal (UQAM), 2008. 
de déterminer les formes de l'éducation qui se pratiquent au sein des musées, mais qui sont trop fréquemment définies en opposition ou en concordance avec certains paramètres scolaires, n'est-il pas envisageable de définir ce qu'est ou n'est pas l'éducation muséale en ellemême ? Un des éléments éclairants provient des diverses propositions de développement de modèles proposés par différents chercheurs qui tentent de cerner et définir la richesse des environnements dans lesquels se déroulent les actions d'éducation en contexte muséal. Parmi ceux-ci, nous en avons relevé quatre principaux - certains provenant de l'Amérique du Nord, un autre de l'Europe - et pour lesquels les grandes orientations seront brièvement présentées afin de dégager quelques axes qui, nous le souhaitons, permettront d'alimenter la réflexion ${ }^{1}$.

\section{Les modèles émergents en éducation muséale}

\section{Le modèle systémique de la situation pédagogique}

Pour le Groupe de recherche sur l'éducation et les musées (GREM) l'éducation muséale s'appuie sur un modèle théorique, soit une représentation abstraite qui explicite les principes, définit les termes, et alimente la réflexion. Le modèle systémique de la situation pédagogique s'inspire des travaux du professeur Renald Legendre. Il permet de dégager le modèle implicite sur lequel se fonde un programme pédagogique ou un programme éducatif muséal et qui met en relation un ensemble de

1 Nous ne prétendons ni à l'exhaustivité ni à la représentativité dans la présentation des modèles. Toutefois, le choix a été privilégié selon l'incidence directe qu'ils ont exercé dans le milieu professionnel de l'éducation muséale. À cet égard, les travaux du Groupe de recherche sur l'éducation et les musées (GREM) de l'UQAM, dirigé par Michel Allard, ont fortement influencé les professionnels de l'éducation principalement dans les musées québécois, mais également en France et en Belgique. Les propositions développées par Georges Hein de l'Exploratorium de San Francisco, ont eu des répercussions directes au sein de l'institution à laquelle il appartient, mais aussi à travers les divers musées, notamment ceux de sciences, aux Etats-Unis. Quant à Falk et Dierking, la légitimité de leurs propositions n'est plus à démontrer puisqu'ils ont été parmi les premiers à analyser la question de l'apprentissage en milieu muséal. Ils ont à travers le temps raffiné leurs modèles et inspiré de nombreux acteurs de la muséologie dans la conception et la réalisation de dispositifs d'exposition ayant un potentiel avéré en éducation. Quant à Eilean Hooper-Greenhill, elle a développé depuis des décénnies une réflexion et une analyse de la dimension éducative des musées en Grande-Bretagne et ses travaux ont mené à la mise en place de guides méthodologiques qui permettent aux professionnels d'évaluer l'ensemble de leurs actions éducatives au sein des musées britanniques. 
composants, soit le sujet (l'élève-visiteur), l'agent (le guide-interprète), l'objet (la thématique) et le milieu (l'institution muséale). Entre ceux-ci se tissent des relations, la relation de support s'articule autour du lien entre l'agent d'éducation et le sujet-visiteur par l'intermédiaire de stratégies et de moyens mis en œuvre. La relation de transposition concerne le lien entre l'agent d'éducation de l'institution muséale et la thématique du programme éducatif. Cette relation se concrétise dans la prise de connaissance du contenu, la définition d'objectifs et la planification de stratégies pédagogiques afin d'adapter la thématique à un public précis. Enfin, la relation d'appropriation définit le lien entre le sujet-visiteur et la thématique; il peut être d'ordre cognitif, affectif, imaginatif ou esthétique. Cette relation se manifeste à trois plans : le savoir, le savoirfaire et le savoir-être ${ }^{1}$. L'appropriation implique la compréhension par le visiteur de la thématique du programme éducatif.

Figure 2

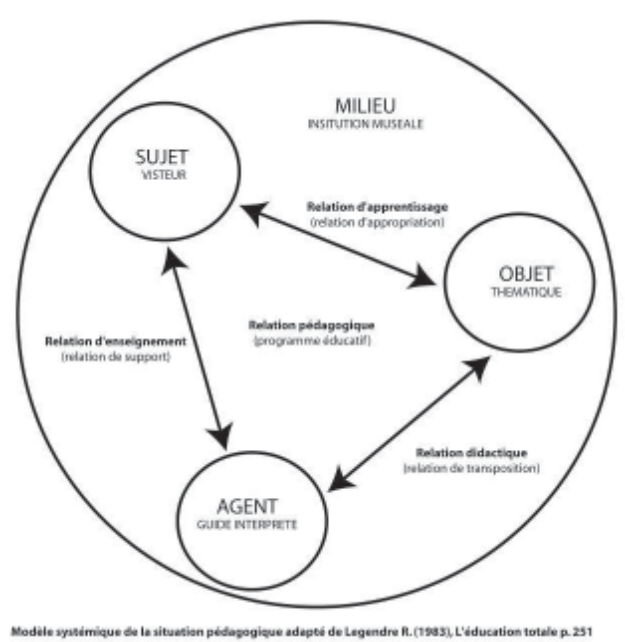

Le modèle didactique d'utilisation des musées à des fins éducatives

Dans le modèle proposé par le GREM, la formulation d'une question constitue la première étape du processus d'apprentissage. L'introduction d'un temps de questionnement à l'école, avant la visite dans le musée permet aux élèves d'observer, de se questionner et le

1 M. Allard et S. BOUCHER, Éduquer au musée, Éditions Hurtubise HMH, Montréal, 1994, 207 p. 
plus possible de développer des hypothèses. Après la visite dans le musée, qui constitue une phase de recherche et de collecte de données, les élèves doivent, à l'école, tirer des conclusions. En analysant et en confrontant les données collectées, les élèves vont pouvoir répondre à leurs questions et vérifier leurs hypothèses. Ce modèle, basé sur un processus intellectuel face à l'objet, comprend trois étapes; trois temps (avant-pendant-après) et deux lieux (école-musée).

Figure 3

\begin{tabular}{|c|c|c|c|c|}
\hline Avant & Ecole & Préparation & interrogation & Questionnement sur l'objet \\
\hline Pendant & Musée & Réalisation & $\begin{array}{l}\text { Cueillete de } \\
\text { données et analyse }\end{array}$ & Observation de l'objet \\
\hline Après & Ecole & Prolongement & $\begin{array}{l}\text { Analyse et } \\
\text { synthese }\end{array}$ & Appropriation de lobjet \\
\hline
\end{tabular}

ALLARD LAROUCHE, LFFEBVRE, MEUNIER VADEBONCOEUR, 1995

\section{Les dimensions de l'éducation muséale}

Le GREM a développé un modèle de pédagogie muséale qui possède fondamentalement une dimension muséologique, une dimension disciplinaire et une dimension sociale. La dimension muséologique réfère naturellement au lieu éducatif particulier qu'est le musée, à l'expérience muséale vécue par le visiteur et aux interactions de ce dernier avec les œuvres et les objets exposés. La dimension disciplinaire rappelle la discipline du musée en adoptant la présentation d'objets de nature artistique, historique, scientifique ou autre. La dimension sociale, quant à elle, correspond aux caractéristiques et aux exigences propres du groupe social auquel appartient le visiteur. Ce groupe peut être la famille, le groupe scolaire, le groupe de loisir ou tout autre groupe organisé composé d'enfants, d'adultes ou d'aînés. 


\section{Figure 4}

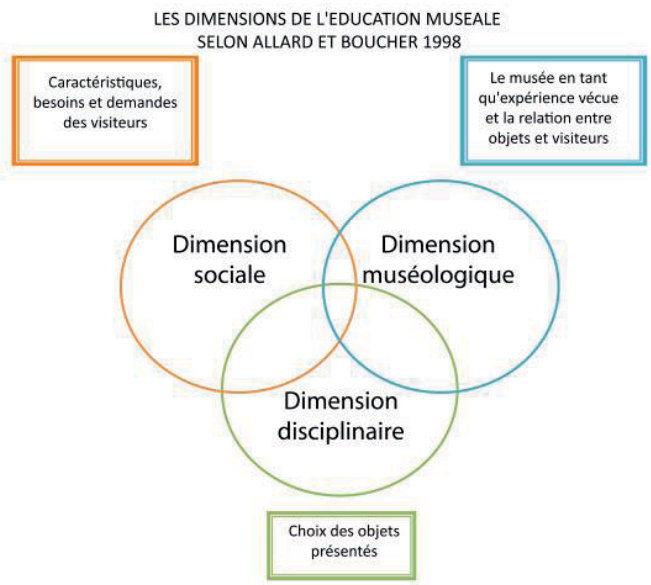

\section{Le modèle constructiviste de l'apprentissage en contexte d'éduca- tion non formelle}

S'il n'est pas le seul à préconiser une approche constructiviste ou socio-constructiviste de l'éducation en contexte muséal, Georges Hein est celui qui développe l'idée que le modèle constructiviste serait le mieux adapté à l'éducation muséale. Il dégage les grandes lignes suivantes de la définition du constructivisme : "Les apprenants construisent eux-mêmes leur savoir - chaque apprenant construit du sens, individuellement (et socialement) - à l'occasion de l' acte d'apprendre "1 . Pour Hein ${ }^{2}$, construire du sens signifie apprendre et cette approche engendre, selon lui, principalement deux choses : premièrement, on doit se centrer sur l'apprenant lorsqu'on réfléchit à l'acte d'apprendre - et non au sujet ou à la leçon à enseigner - et, deuxièmement, il n'y a pas de savoir qui soit indépendant de la signification que l'apprenant, ou que la communauté d'apprenants, prête à l'expérience (construite). Ce chercheur admet qu'il n'existe rien de tel qu'un savoir " externe " distinct de l'apprenant, mais uniquement un savoir que nous construisons nous-mêmes et qui est le résultat de l'apprentissage. Il dégage une série de principes relatifs à l'appren-

1 Tiré de la communication de G.HEIN, Committee for Education and Cultural Action/ ICOM-CECA Conference, Jérusalem 1991, traduit de l'anglais par G. Fritsch, URL : http://www.exploratorium.edu/IFI/resources/constructivistlearning.html

2 G.E. HEIN, Learning in the Museum, Routledge, 1998, 203 p. 
tissage en contexte muséal selon une perspective constructiviste. Parmi ceux-ci, nous en retenons quelques uns et présentons leurs diverses implications en éducation muséale ${ }^{1}$.

Selon Hein, apprendre est un processus actif dans lequel l'apprenant se sert des données sensorielles pour construire du sens. Ce principe est largement appliqué dans les musées. D'une part, par les contextes souvent riches et variés en stilumis que le musée et les expositions proposent aux visiteurs et, d'autre part, par les formes variées de présentation d'un même contenu (objets, textes, sources iconographiques, autres). Hein soutient aussi qu'apprendre est une activité sociale et que notre façon d'apprendre est intimement liée à nos relations avec les autres, nos éducateurs, nos pairs, notre famille. En contexte muséal, il s'agit des personnes qui nous accompagnent ou nous entourent dans une exposition. Les musées, nous dit Hein, sont des lieux conviviaux où la visite se déroule la plupart du temps en groupe et ils devraient être des lieux propices à la conversation, à l'échange, au débat. À cet égard, plusieurs chercheurs ont démontré que la dimension sociale de la visite en contexte muséal est l'un des facteurs déterminants de l'apprentissage. $^{2}$

Hein maintient qu'apprendre nécessite du temps et ne se fait ni spontanément, ni instantanément. Il ne croit pas que cela puisse se faire dans les cinq à dix minutes que l'on passe habituellement dans une salle d'exposition. À ce propos, Hein suggère des modes d'appropriation d'une exposition qui soient ponctuées dans le temps. Par exemple, les groupes scolaires - ou toute autre catégorie de visiteurs - pourraient passer plus d'une heure dans un musée et la proposition de développer des projets qui les incitent à demeurer dans le musée plus longtemps - plusieurs heures, à répétition, quelques jours - est, selon lui, à préconiser. Afin de favoriser des contextes d'apprentissage riches au sein des musées et des expositions, Hein propose de contextualiser les situations d'apprentissage, de mettre en place des contextes permettant de bâtir de nouvelles connaissances à partir de connaissances déjà acquises et de susciter et maintenir la motivation des visiteurs qui constitue un élément clé de l'apprentissage.

1 Pour une liste complète des principes proposés par G. HEIN, consulter http://www. exploratorium.edu/IFI/resources/constructivistlearning.html

2 Voir à ce sujet, entre autres, L. DIERKING, " Rôle de l'interaction sociale dans l'expérience muséale ", in Publics et Musées, 5, 1995, pp. 19-43. 
Le modèle constructiviste tel que définit par Hein se divise en deux principaux domaines : les théories dites de l'apprentissage et les théories dites du savoir (de la connaissance). Il place l'apprentissage sur un axe allant de l'apprentissage passif selon lequel l'apprenant reçoit les contenus d'apprentissage par des approches directives; à l'apprentissage actif, selon lequel l'apprenant détient un rôle actif dans le processus même d'apprentissage. En plaçant ces deux théories sur deux axes différents, il délimite des cadres et des modèles d'apprentissages qui laissent une autonomie variable à l'apprenant dans sa propre démarche.

\section{Figure 5}

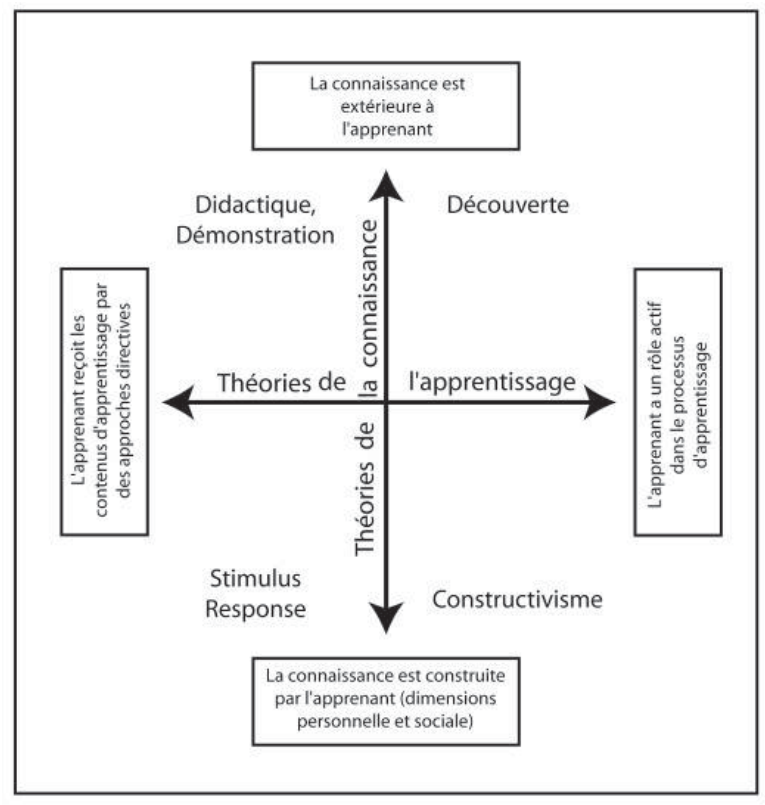

Georges Hein, 1991

Dans ce schéma, le modèle behavioriste (stimuli-réponse) qui est positionné à gauche, est assez peu adapté aux musées selon Hein, en ce sens que l'apprenant n'est pas directement partie prenante de son processus d'apprentissage. Il ne fait que répondre à une proposition extérieure qui lui est formulée. Aussi, il suggère que l'éducation muséale se situe plutôt dans la partie droite du schéma, dans une perspective de découverte qui renvoie à la conception de l'éducation de Dewey et de la construction sociale des apprentissages qui correspond aux théories piagétienne et vigotskienne. 


\section{Le modèle contextuel de l'apprentissage et le modèle de l'expé- rience interactive}

Pour leur part, les modèles de Falk et Dierking proposent une représentation de la situation d'apprentissage en milieu muséal. Du point de vue du "visiteur-apprenant", la situation d'apprentissage est déterminée selon trois contextes : physique, personnel et social. Pour définir l'apprentissage en milieu muséal, les auteurs ont recours au terme "free-choice learning " - apprentissage libre-choix -. Il ressort de cette expression, l'interaction de l'individu avec son environnement. Les auteurs définissent l'apprentissage libre-choix comme " [...] l'apprentissage que font les gens lorsqu'ils contrôlent où, quoi, quand et avec qui ils apprennent ". ${ }^{1}$ Ils définissent l'apprentissage comme " $[\ldots]$ un processus continu d'assimilation et d'aménagement de l'information par le biais du contexte social, du contexte physique et du contexte psychologique"2.

Le modèle contextuel de l'apprentissage ${ }^{3}$ implique l'intervention de trois contextes : physique, social, personnel : “[...] l'expérience du visiteur au musée n'est pas simplement le résultat de son interaction avec les expositions, il est plutôt la somme du contexte personnel, du contexte social et du contexte physique de l'individu." ${ }^{4}$ Les auteurs expliquent le rôle des différents contextes de ce modèle, à savoir que toute forme d'apprentissage trouve son début et sa fin dans les intérêts, les motivations, les connaissances préalables et les expériences de l'individu ; que pourquoi et ce que l'individu apprend est intimement lié au contexte social, au contexte culturel et au contexte historique et que l'apprentissage est à la fois une expérience individuelle et une expérience collective ; que l'apprentissage est favorisé par un contexte

1 J.H. FALK et L.D. DIERKING, Lessons without Limit, New York, Altamira, 2002, p. 6. Traduction libre de " $[. .$.$] learning people do when they get to control what to$ learn, when to learn, where to learn, and with whom to learn ".

2 J.H. FALK et L.D. DIERKING, The Museum Experience, Washington, Whalesback, 1992, p. 113. Traduction libre de " $[. .$.$] a continuous, active process of assimilating and$ accommodating information with social, physical, and psychological contexts."

3 Ibid p. 55. Traduction libre de " $[\ldots]$ the visitor's museum experience is not just the result of interactions with exhibits, but the sum of his constructed personal, social, and physical contexts."

4 Ibid p. 55. Traduction libre de " $[\ldots]$ the visitor's museum experience is not just the result of interactions with exhibits, but the sum of his constructed personal, social, and physical contexts." 
physique approprié, l'aménagement de l'espace et le design de l'exposition ont une incidence directe sur l'apprentissage et que l'apprentissage est influencé par le stade de développement de l'individu. ${ }^{1}$

Figure 6

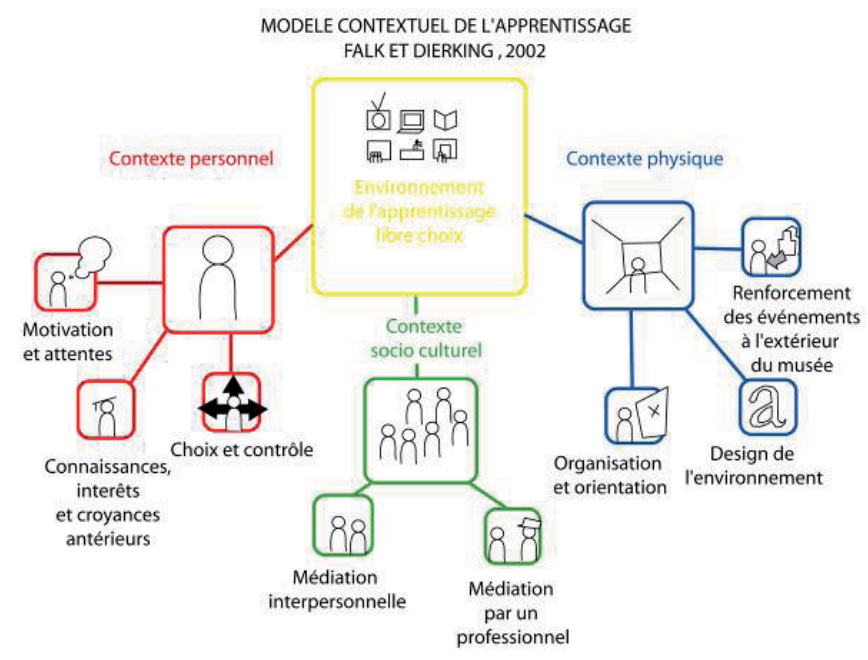

Le modèle de l'expérience interactive présenté dans leur première publication, The Museum Experience reprend les trois contextes (personnel, social et physique) chers à Falk et Dierking. Ces contextes déterminent quand, quoi, pourquoi et comment l'individu apprend dans un environnement d'“apprentissage libre-choix". L'intersection des trois contextes forme : l'expérience interactive ${ }^{2}$.

1 J.H. FALK et L.D. DIERKING, Lessons without Limit, New York, Altamira, 2002, p 56. Traduction libre de " All learning begins, and ends with the individual's unique interests, motivations, prior knowledge, and experience. Learning is both an individual experience and a group experience. What someone learns learns, let alone why someone learns, is inextricably bound to the social, cultural, and historical context in which that learning takes place. Learning is facilitated by appropriate physical contexts and by well thought-out and built designs - the outdoors or an immersive zoo for learning about animals, an art museum or studio for learning about the visual arts, a historic site or reenactment for learning about history. Learning is influenced by the developmental stage that the person is at."

2 L'expression " expérience interactive" est une traduction libre de "interactive 
Selon Falk et Dierking, "La décision de visiter un musée soustend une intention de jumeler des intérêts personnels à des intérêts sociaux au sein d'un contexte physique [...] ". ${ }^{1}$ Ils expliquent ainsi que l'expérience se " $[\ldots]$ déroule dans un contexte physique comprenant une architecture et des objets que nous appelons le musée. Dans cet endroit, l'individu perçoit le monde à travers son contexte personnel. Il partage ses expériences avec les gens qui l'entourent possédant leur propre contexte personnel. Le regroupement de ces expériences crée le contexte social. "2 Les chercheurs précisent que chaque contexte revêt une importance dans l'expérience interactive du musée mais qu'ils ne sont pas nécessairement sollicités de manière proportionnelle. De plus, même s'ils sont présentés comme des entités séparées, ils interagissent selon un tout ${ }^{3}$.

\section{Figure 7}

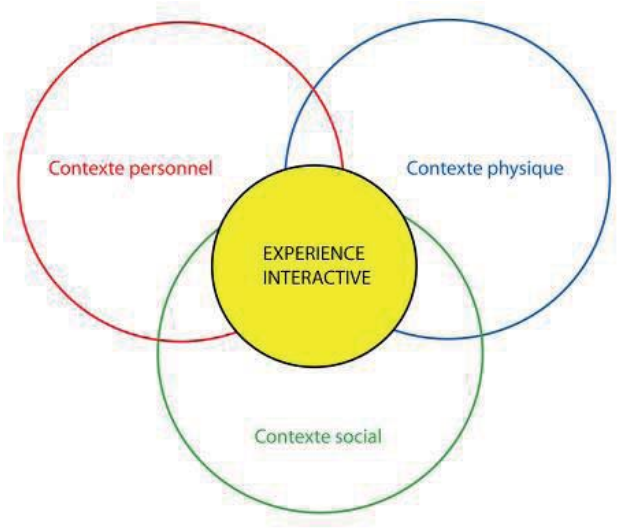

MODELE DE L'EXPERIENCE INTERACTIVE, FALK AND DIERKING , 1992

experience".

1 J.H. FALK et L.D. DIERKING, The Museum Experience, Washington, Whalesback, 1992, p. 12. Traduction libre de "The decision to visit a museum involves matching personal and social interests and desires with the anticipated physical context $[\ldots] "$.

2 Ibid p. 4. Traduction libre de " [...] occurs within the physical context, a collection of structures and things we call the museum. Within the museum is the visitor, who perceives the world through his own personal context. Sharing this experience are various other people, each with their own personal context, which together create a social context."

3 Ibid p. 7. Traduction libre de " $[\ldots]$ although the three contexts can be viewed separately, they function, in fact, as an integrated whole." 


\section{La dimension temporelle du modèle contextuel de l'apprentissage}

Selon Falk et Dierking, "L'apprentissage ainsi que ses composants sont éphémères, ils changent constamment. L'apprentissage peut donc être considéré comme un amalgame infini des trois contextes à travers le temps dans le but de créer du sens. " Les visiteurs " [...] assimilent ce qu'ils ont observé dans le musée par le truchement de catégories cognitives de nature individuelle, qui sont déterminées par des événements personnels avant et après la visite du musée. "2 D'après ces chercheurs, un regard instantané ou une mesure ponctuelle de l'expérience d'apprentissage est inadéquat puisque celui-ci ne se termine pas lorsque l'individu quitte le musée. Il se poursuit dans le temps, pendant une période indéterminée. Il faut donc prendre en compte l'existence d'un passé, d'un présent et d'un futur dans l'expérience d'apprentissage en contexte muséal.

\section{Figure 8}

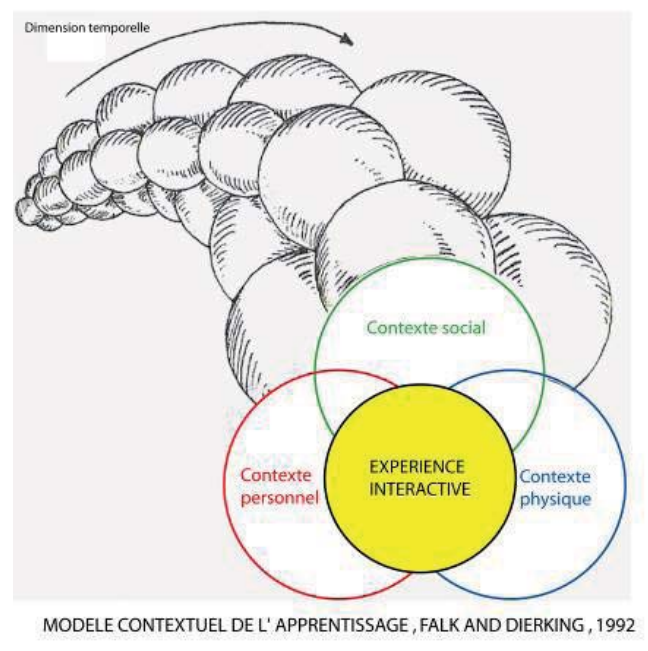

1 J.H. FALK et L.D. DIERKING, 2000. Learning from Museums: Visitor Experiences and the Making of Meaning. Walnut creek: Altamira-Press.p. 11. Traduction libre de "Learning, as well as its constituent pieces, is ephemeral, always changing. Ultimately, then, learning can be viewed as the never-ending integration of these three contexts over time in order to make meaning."

2 J.H. FALK et L.D. DIERKING, op. cit., p. 123. Traduction libre de “ [...] assimilate events and observations in mental categories of personal significance and character, determined by events in their lives before and after a museum visit." 


\section{Le modèle centré sur l'apprentissage à l'aide de l'objet}

L'approche d'Hooper-Greenhill rejoint en tous points celle du canadien Duncan Cameron qui soutient que le musée est un système de communication dont la spécificité s'appuie sur le travail réalisé avec et autour des objets (artefacts, manetifacts et kinetifacts), ce qu'il nomme les " vraies choses ". À l'instar de Cameron, Hooper-Greenhill met de l'avant que "L'éducation muséale est concernée par l'enseignement et l'apprentissage à l'aide des objets et des spécimens ". Comment enseigne-t-on et comment apprend-t-on avec et à partir des objets ? Sa démarche suggère un questionnement à partir de l'objet. Le modèle qu'elle propose s'adapte tant à l'école qu'au musée. Selon elle, l'éducation par les objets permet l'atteinte de plusieurs compétences, notamment l'éveil des sens, le développement de la pensée et du langage. Son modèle se résume principalement en trois étapes de travail et d'étude autour de l'objet. L'étape un consiste à développer et atteindre une perception sensorielle afin d'accumuler le plus de données possible quant à l'objet à l'étude. L'étape deux propose d'engager une discussion à partir des données recueillies, de les relier à des informations et des expériences antérieures, et de les comparer avec les perceptions des autres visiteurs. L'étape trois vise la construction d'hypothèses. Hooper-Greenhill soutient que les interprétations personnelles comptent autant que les interprétations " scolaires " et incite à cette étape de dégager des hypothèses et de déduire un ensemble de propositions fondées sur les deux étapes précédentes.

\section{Figure 9}

\begin{tabular}{|l|l|l|}
\hline Étape 1 & Perception sensorielle & Collecte de données à propos de l'objet \\
\hline Étape 2 & Discussion et analyse & $\begin{array}{l}\text { Discussions autour des données collectées } \\
\text { Les données sont reliées à des informations antérieures } \\
\text { et comparées avec les perceptions des autres visiteurs }\end{array}$ \\
\hline Étape 3 & $\begin{array}{l}\text { Comparaison et } \\
\text { synthèse }\end{array}$ & Constructions d'hypothèses \\
\hline
\end{tabular}

1 D.F. CAMERON, 1968, “ Un point de vue : le musée considéré comme système de communication et les implications de ce système pour les programmes éducatifs muséaux ”, in Vagues, 1992, pp. 271-280.

2 E. HOOPER-GREENHILL, The Educational role of the museum, London, Routledge, 1994, p. 229. Traduction libre de "Museum education is centrally concerned with teaching from and learning with objects and specimens". 
Modèle à trois étapes de travail et d'étude autour de l'objet proposé par Hopper-Greenhill :

Figure 10

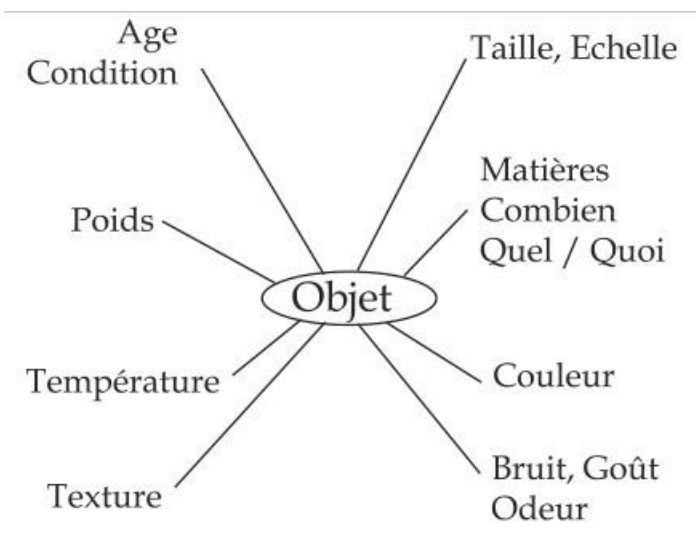

L'exploration sensorielle est l'une des premières approches pour travailler avec des objets Eilean Hooper Greenhill, 1994

Figure 11

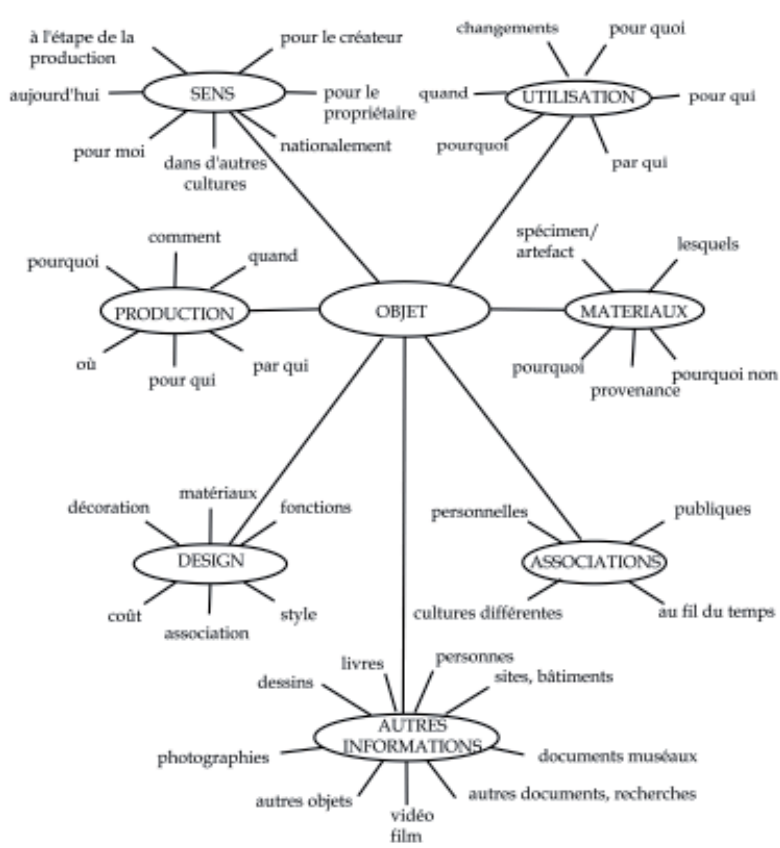

Discussion et analyse, Eilean Hooper Greenhill 
Figure 12

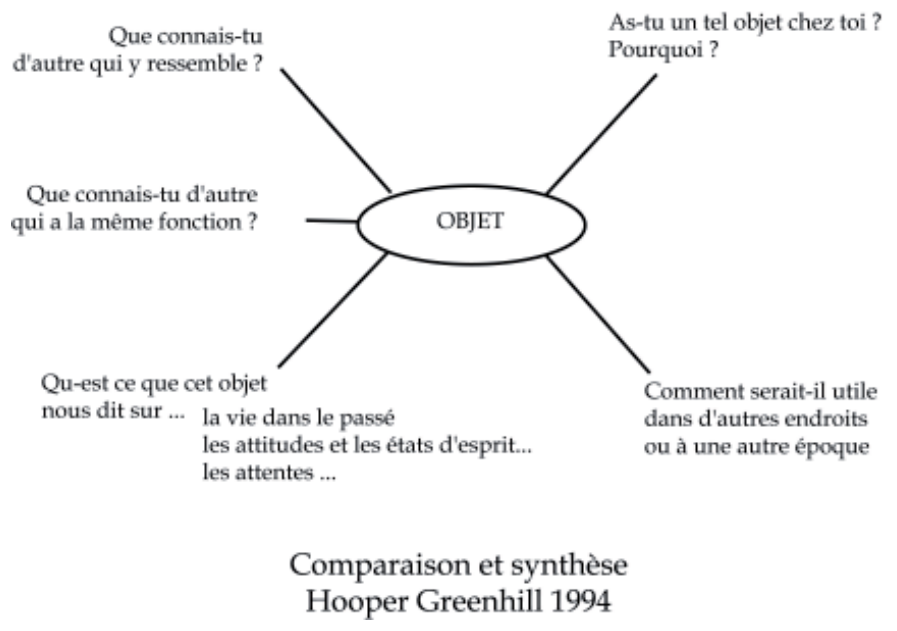

\section{L'éducation muséale et les configurations du savoir}

L'étude des fonctions au sein du musée et des modèles émergeants en éducation muséale révèle un ensemble d'informations quant à l'histoire des musées, mais elle n'en est certes pas l'unique entrée. En effet, plutôt que de cerner les spécificités ou les similarités de ces institutions, Foucault ${ }^{1}$ suggère de décrire les discontinuités, les ruptures, les déplacements, les dispersions, voire les tensions au sein et entre les institutions muséales. À cet égard, toujours selon Foucault, une histoire " effective " doit savoir décrire les pratiques, ainsi que les conditions qui rendent ces pratiques acceptables dans une société donnée, à une époque donnée. Les structures de la connaissance sont sous l'influence de certains facteurs spécifiques : culturels, sociaux, politiques ou scientifiques. Ces facteurs interagissent étroitement : ils travaillent en synergie ou en tension ; ensemble ou séparément ; les uns avec ou contre les autres dans un état de flux constant qui fait en sorte que la connaissance est en perpétuelle et constante redéfinition. À travers ce flux de transformations rapides, et parfois contradictoires, il est possible de faire ressortir des courants dominants dans l'activité intellectuelle de certaines époques. Dans son ouvrage L'Ordre des choses, Foucault ${ }^{2}$ s'est appliqué à décrire les épistèmes, —ou grands courants— qui ont

1 M. FOUCAULT, L'Ordre des choses, 1970.

2 Ibid. 
dominé l'activité intellectuelle, de la Renaissance à l'époque moderne. Il a ainsi identifié trois grands ensembles de connaissances réglées et propres à un groupe social, à une époque : l'épistème de la Renaissance, l'épistème classique et l'épistème moderne séparés entre eux par d'importantes périodes de tension, de perturbation et de rupture qui ont induit le passage d'un grand courant à l'autre.

L'épistème de la Renaissance correspond à la période à laquelle on accède à la connaissance par des méthodes d'interprétation qui révèlent des similitudes entre les objets. Les relations entre les objets sont infinies et il n'y a pas de limites à l'accumulation des configurations qui sont toutes interdépendantes les unes des autres. Le monde et toutes les choses qu'il contient sont reliés entre eux, de manière infinie et continue, par de nombreux liens souvent demeurés cachés. L'épistème classique découle de l'importante rupture qui survient au début du XVII siècle. Les bases de la structure de la connaissance se transforment : on ne découvre plus la vérité en cherchant les ressemblances, on cherche plutôt à décrire les différences. Toutes les choses matérielles, mesurables et visibles sont nommées, caractérisées et classifiées. L'homme classique privilégie l'ordre, la mesure, la série, la hiérarchie. L'intention visée consiste alors à pouvoir placer toutes les représentations (choses) du monde selon une grande table de classification universelle, immuable et vraie, représentative du monde en son entier. L'épistème moderne correspond à une autre grande rupture avec l'époque précédente. À la fin du XVIII ${ }^{\mathrm{e}}$ siècle, la connaissance ne repose désormais ni sur les similitudes, ni sur les différences trouvées dans l'aspect visible des choses. Le monde est plutôt composé de structures organiques qui se manifestent par des relations internes multiples remplissant des fonctions particulières. La compréhension de l'objet ne passe plus par la description de ce qui est visible à sa surface. L'important est de découvrir pourquoi les choses en sont venues à être telles qu'elles sont. On cherche des analogies de fonctions et de similitudes dans les relations établies qui surviennent entre les parties internes. C'est à cette époque que la philosophie, les sciences humaines et les sciences sociales naissent et que l'histoire naturelle cède la place à la biologie.

Des recherches ont montré que l'évolution des musées, de leurs politiques de collection et de conservation, de même que la présentation de leurs collections par la mise en espace et la muséographie, s'est 
développée en parallèle de l'évolution des configurations qu'empruntent les formes de savoir. Plusieurs muséologues ont utilisé cette théorie afin d'expliquer l'histoire des musées et de les définir en tant qu'institution. L'expression des épistèmes de Foucault au sein des musées permet de porter un regard sur les configurations du savoir au sein de ces institutions. Au premier épistème, celui de la Renaissance, correspond le temps des cabinets de curiosités. Les objets collectionnés sont disposés en fonction d'un rang, d'une place, qu'on leur assigne, dans l'ordre symbolique et secret du "grand théâtre du monde". À l'époque classique, temps du deuxième épistème, c'est l'avènement du cartésianisme et de l'analyse objective. Les objets des collections sont classés, ordonnés, hiérarchisés. Au troisième épistème, celui de l'époque moderne, correspond la période de la naissance des grands musées publics. C'est le siècle des Lumières et les musées se font encyclopédie, ils deviennent les " narrateurs de l'aventure humaine". Les discours des musées sont centrés sur des idéaux à atteindre. Aux trois épistèmes décrits par Foucault s'ajoute aujourd'hui un quatrième épistème né d'une rupture dans la structure de la connaissance humaine survenue lors de l'apparition des sociétés dites postindustrielles. À la fin des années 1970, Lyotard décrit les caractéristiques propres à la société postmoderne ${ }^{1}$. C'est le règne des libertés individuelles : la conscience est individuelle, les choix sont personnels et subjectifs, le relativisme est intégral. Dans ce contexte, les musées qui avaient acquis une certaine légitimité institutionnelle, ne sont plus habilités à énoncer des idéaux, ni à prôner une direction commune. Les musées de l'époque post-moderne deviennent moins dogmatiques. Ils ne présentent plus un savoir encyclopédique, ordonné et considéré comme immuable. Ils se transforment et engagent un dialogue avec leur collectivité. Ils présentent des " points de vue ", souvent formulés hors de l'institution, et invite le visiteur à participer activement à la production de sens. Les grandes représentations communes sont maintenant des morceaux épars de passé, de présent et de vérité. Dans l'ère postmoderne, la visite dans un musée s'inscrit surtout dans un mode de loisir. Suivant cette logique, le visiteur devient un " client" qui s'approprie un " produit culturel " mis en marché par une organisation qui poursuit des objectifs de performance économique et qui entre en compétition avec l'ensemble des industries culturelles. Ainsi, les expositions temporaires sont privilégiées, elles sont transi-

1 J.-F. LYOTARD, La condition postmoderne, Rapport sur le savoir, Paris, Les Éditions de Minuit, 1979. 
toires, comparativement aux expositions permanentes. Bien qu'elles aient l'avantage de pouvoir montrer une hétérogénéité d'expériences, de cultures, d'idées et de points de vue, les expositions temporaires n'obéissent pas toujours à ces possibilités et demeurent souvent très conservatrices ne prenant que peu de risques dans le développement des contenus et des propos qu'elles présentent.

Une brève analyse du rapport au savoir à partir de la théorie de Foucault et des quatre modèles en éducation muséale permet de dégager quelques constats qui témoignent d'orientations éducatives du musée. D'abord, l'ensemble des chercheurs préconise des approches par lesquelles le contact et le travail à partir des objets —au sens largeregroupés dans un contexte muséal est central. De plus, le visiteur quel qu'il soit est généralement toujours pris en compte. Enfin, les contextes singuliers dans lesquels les visiteurs sont appelés à déambuler et interagir revêtent eux aussi une importance dans la mission éducative du musée. Déjà, Allard et Boucher ${ }^{1}$ avaient proposé des paradigmes relatifs à l'éducation muséale. Il s'agit de l'éducation centrée sur l'objet muséal (paradigme rationnel); l'éducation centrée sur le message transmis entre autres par le biais de l'objet muséal (paradigme technologique); l'éducation sans objet centrée sur le milieu (paradigme inventif); l'éducation centrée sur le visiteur (paradigme humaniste). Cette catégorisation a pour intérêt de proposer les bases sur lesquelles développer des orientations et des positions du contexte dans lequel se développe le savoir au sein des musées. Il serait pertinent d'établir une mise en relation de ces paradigmes avec les modèles émergeants en éducation muséale afin de les situer quant au rapport au savoir qu'ils développent et incidemment, proposent à leurs publics.

1 M. ALLARD et S. BOUCHER, op. cit.. 